\title{
Vertex Component Analysis: A Fast Algorithm to Unmix Hyperspectral Data
}

\author{
José M. P. Nascimento, Student Member, IEEE, and José M. Bioucas Dias, Member, IEEE
}

\begin{abstract}
Given a set of mixed spectral (multispectral or hyperspectral) vectors, linear spectral mixture analysis, or linear unmixing, aims at estimating the number of reference substances, also called endmembers, their spectral signatures, and their abundance fractions. This paper presents a new method for unsupervised endmember extraction from hyperspectral data, termed vertex component analysis (VCA). The algorithm exploits two facts: 1) the endmembers are the vertices of a simplex and 2) the affine transformation of a simplex is also a simplex. In a series of experiments using simulated and real data, the VCA algorithm competes with state-of-the-art methods, with a computational complexity between one and two orders of magnitude lower than the best available method.
\end{abstract}

Index Terms-Linear unmixing, simplex, spectral mixture model, unmixing hypespectral data, unsupervised endmember extraction, vertex component analysis (VCA).

\section{INTRODUCTION}

$\mathbf{H}$ YPERSPECTRAL remote sensing exploits the electromagnetic (EM) scattering patterns of different materials at specific wavelengths [1], [2]. Hyperspectral sensors have been developed to sample the scattered portion of the EM spectrum extending from the visible region through the near-infrared and midinfrared, in hundreds of narrow contiguous bands [3], [4]. The number and variety of potential civilian and military applications of hyperspectral remote sensing is enormous [5], [6].

Very often, the resolution cell corresponding to a single pixel in an image contains several substances (endmembers) [3]. In this situation, the scattered energy is a mixing of the endmember spectra. A challenging task underlying many hyperspectral imagery applications is then decomposing a mixed pixel into a collection of reflectance spectra, called endmember signatures, and the corresponding abundance fractions [7]-[9].

Depending on the mixing scales at each pixel, the observed mixture is either linear or nonlinear [10], [11]. A linear mixing model holds approximately when the mixing scale is macroscopic [12] and there is negligible interaction among distinct endmembers [2], [13]. If, however, the mixing scale is microscopic (or intimate mixtures) [14], [15] and the incident solar

Manuscript received January 6, 2004; revised December 21, 2004. This work was supported in part by the Fundação para a Ciência e Tecnologia under the Projects POSI/34071/CPS/2000 and PDCTE/CPS/49967/2003 and in part by the Departamento de Engenharia de Electrónica e Telecomunicações e de Computadores of the Instituto Superior de Engenharia de Lisboa.

J. M. P. Nascimento is with the Instituto Superior de Engenharia de Lisboa and the Instituto de Telecomunicações, 1949-001 Lisbon, Portugal (e-mail: zen@isel.pt).

J. M. Bioucas Dias is with the Instituto de Telecomunicações and the Instituto Superiror Técnico, 1949-001 Lisbon, Portugal (e-mail: bioucas@1x.it.pt).

Digital Object Identifier 10.1109/TGRS.2005.844293 radiation is scattered by the scene through multiple bounces involving several endmembers [16], the linear model is no longer accurate.

Linear spectral unmixing has been intensively researched in the last years [8], [9], [11], [17]-[20]. It considers that a mixed pixel is a linear combination of endmember signatures weighted by the correspondent abundance fractions. Under this model, and assuming that the number of substances and their reflectance spectra are known, hyperspectral unmixing is a linear problem for which many solutions have been proposed (e.g., maximum-likelihood estimation [7], spectral signature matching [21], spectral angle mapper [22], subspace projection methods [23], [24], and constrained least squares [25]).

In most cases, the number of substances and their reflectances are not known and, then, hyperspectral unmixing falls into the class of blind source separation problems [26]. independent component analysis (ICA) has recently been proposed as a tool to blindly unmix hyperspectral data [27]-[30]. ICA is based on the assumption of mutually independent sources (abundance fractions), which is not the case of hyperspectral data, since the sum of abundance fractions is constant, implying statistical dependence among them. This dependence compromises ICA applicability to hyperspectral images as shown in [20] and [31]. In fact, ICA finds the endmember signatures by multiplying the spectral vectors with an unmixing matrix which minimizes the mutual information among channels. If sources are independent, ICA provides the correct unmixing, since the minimum of the mutual information corresponds to and only to independent sources. This is no longer true for dependent fractional abundances. Nevertheless, some endmembers may be approximately unmixed. These aspects are addressed in [31].

Under the linear mixing model, the observations from a scene are in a simplex whose vertices correspond to the endmembers. Several approaches [32]-[34] have exploited this geometric feature of hyperspectral mixtures [33].

The minimum volume transform (MVT) algorithm [34] determines the simplex of minimum volume containing the data. The method presented in [35] is also of MVT type, but by introducing the notion of bundles, it takes into account the endmember variability usually present in hyperspectral mixtures.

The MVT type approaches are complex from the computational point of view. Usually, these algorithms first find the convex hull defined by the observed data and then fit a minimum volume simplex to it. For example, the gift wrapping algorithm [36] computes the convex hull of $n$ data points in a $d$-dimensional space with a computational complexity of $O\left(n^{\lfloor d / 2\rfloor+1}\right)$, where $\lfloor x\rfloor$ is the highest integer lower or equal than $x$, and $n$ is the number of samples. The complexity of the method presented 
in [35] is even higher, since the temperature of the simulated annealing algorithm therein used shall follow a $\log (\cdot)$ law [37] to assure convergence (in probability) to the desired solution.

Aiming at a lower computational complexity, some algorithms such as the pixel purity index (PPI) [33] and the N-FINDR [38] still find the minimum volume simplex containing the data cloud, but they assume the presence in the data of at least one pure pixel of each endmember. This is a strong requisite that may not hold in some datasets. In any case, these algorithms find the set of most pure pixels in the data.

The PPI algorithm uses the minimum-noise fraction (MNF) [39] as a preprocessing step to reduce dimensionality and to improve the signal-to-noise ratio (SNR). The algorithm then projects every spectral vector onto skewers (large number of random vectors) [33], [40], [41]. The points corresponding to extremes, for each skewer direction, are stored. A cumulative account records the number of times each pixel (i.e., a given spectral vector) is found to be an extreme. The pixels with the highest scores are the purest ones.

The N-FINDR algorithm [38] is based on the fact that in $p$ spectral dimensions, the $p$-volume defined by a simplex formed by the purest pixels is larger than any other volume defined by any other combination of pixels. This algorithm finds the set of pixels defining the largest volume by inflating a simplex inside the data.

ORASIS [42], [43] is a hyperspectral framework developed by the Naval Research Laboratory consisting of several algorithms organized in six modules: exemplar selector, adaptative learner, demixer, knowledge base/spectral library, and spatial postprocessor. The first step consists in flat fielding the spectra. Next, the exemplar selection module is used to select spectral vectors that best represent the smaller convex cone containing the data. The other pixels are rejected when the spectral angle distance is less than a given threshold. The procedure finds the basis for a subspace of a lower dimension using a modified Gram-Schmidt orthogonalization. The selected vectors are then projected onto this subspace, and a simplex is found by an MVT process. ORASIS is oriented to real-time target detection from uncrewed air vehicles using hyperspectral data [44].

In this paper we introduce the vertex component analysis (VCA) algorithm to unmix linear mixtures of endmember spectra. The algorithm is unsupervised and exploits two facts: 1) the endmembers are the vertices of a simplex and 2) the affine transformation of a simplex is also a simplex. It works with unprojected and with projected data. As PPI and N-FINDR algorithms, VCA also assumes the presence of pure pixels in the data. The algorithm iteratively projects data onto a direction orthogonal to the subspace spanned by the endmembers already determined. The new endmember signature corresponds to the extreme of the projection. The algorithm iterates until all endmembers are exhausted. VCA performs much better than PPI and better than or comparable to N-FINDR; yet it has a computational complexity between one and two orders of magnitude lower than N-FINDR.

The paper is structured as follows. Section II describes the geometric fundamentals of the proposed method. Sections III and IV evaluate the proposed algorithm using simulated and real data, respectively. Section $V$ ends the paper by presenting some concluding remarks.

\section{Vertex COMponent Analysis Algorithm}

Assuming the linear mixing scenario, each observed spectral vector is given by

$$
\mathbf{r}=\mathbf{x}+\mathbf{n}=\mathbf{M} \underbrace{\gamma \boldsymbol{\alpha}}_{\mathbf{s}}+\mathbf{n}
$$

where $\mathbf{r}$ is an $L$-vector ( $L$ is the number of bands), $\mathbf{M} \equiv\left[\mathbf{m}_{1}, \mathbf{m}_{2}, \ldots, \mathbf{m}_{p}\right]$ is the mixing matrix $\left(\mathbf{m}_{i}\right.$ denotes the $i$ th endmember signature and $p$ is the number of endmembers present in the covered area), $\mathbf{s} \equiv \gamma \boldsymbol{\alpha}$ ( $\gamma$ is a scale factor modeling illumination variability due to surface topography), $\boldsymbol{\alpha}=\left[\alpha_{1}, \alpha_{2}, \ldots, \alpha_{p}\right]^{T}$ is the abundance vector containing the fractions of each endmember (the notation $(\cdot)^{T}$ stands for vector transposed) and $\mathbf{n}$ models system additive noise.

Owing to physical constraints [19], abundance fractions are nonnegative $(\boldsymbol{\alpha} \succeq 0)$ and satisfy the so-called positivity constraint $\mathbf{1}^{T} \boldsymbol{\alpha}=1$, where 1 is a $p \times 1$ vector of ones. Each pixel can be viewed as a vector in an $L$-dimensional Euclidean space, where each channel is assigned to one axis of space. Since the set $\left\{\boldsymbol{\alpha} \in \Re^{p}: \mathbf{1}^{T} \boldsymbol{\alpha}=1, \boldsymbol{\alpha} \succeq 0\right\}$ is a simplex, then the set $S_{x}=\left\{\mathbf{x} \in \Re^{L}: \mathbf{x}=\mathbf{M} \boldsymbol{\alpha}, \mathbf{1}^{T} \boldsymbol{\alpha}=1, \boldsymbol{\alpha} \succeq 0\right\}$ is also a simplex. However, even assuming $\mathbf{n}=\mathbf{0}$, the observed vector set belongs to $C_{p}=\left\{\mathbf{r} \in \Re^{L}: \mathbf{r}=\mathbf{M} \gamma \boldsymbol{\alpha}, \mathbf{1}^{T} \boldsymbol{\alpha}=1, \boldsymbol{\alpha} \succeq 0, \gamma \geq 0\right\}$ that is a convex cone, owing to scale factor $\gamma$. Fig. 1(a) illustrates a simplex and a cone, projected on a two-dimensional subspace, defined by a mixture of three endmembers. The simplex boundary is a triangle whose vertices correspond to the endmembers shown in Fig. 2. Small and medium dots are simulated mixed spectra belonging to the simplex $S_{x}(\gamma=1)$ and to the cone $C_{p}(\gamma>0)$, respectively.

The projective projection of the convex cone $C_{p}$ onto a properly chosen hyperplane is a simplex with vertices corresponding to the vertices of the simplex $S_{x}$. This is illustrated in Fig. 1(b). The simplex $S_{p}=\left\{\mathbf{y} \in \Re^{L}: \mathbf{y}=\mathbf{r} /\left(\mathbf{r}^{T} \mathbf{u}\right), \mathbf{r} \in C_{p}\right\}$ is the projective projection of the convex cone $C_{p}$ onto the plane $\mathbf{r}^{T} \mathbf{u}=1$, where the choice of $\mathbf{u}$ assures that there is no observed vectors orthogonal to it.

After identifying $S_{p}$, the VCA algorithm iteratively projects data onto a direction orthogonal to the subspace spanned by the endmembers already determined. The new endmember signature corresponds to the extreme of the projection. Fig. 1(b) illustrates the two iterations of VCA algorithm applied to the simplex $S_{p}$ defined by the mixture of two endmembers. In the first iteration, data are projected onto the first direction $\mathbf{f}_{1}$. The extreme of the projection corresponds to endmember $\mathbf{m}_{a}$. In the next iteration, endmember $\mathbf{m}_{b}$ is found by projecting data onto direction $\mathbf{f}_{2}$, which is orthogonal to $\mathbf{m}_{a}$. The algorithm iterates until the number of endmembers is exhausted.

\section{A. Dimensionality Reduction}

Under the linear observation model, spectral vectors are in a subspace of dimension $p$. If $p \ll L$, it is worthy to project the observed spectral vectors onto the subspace signal. This leads 


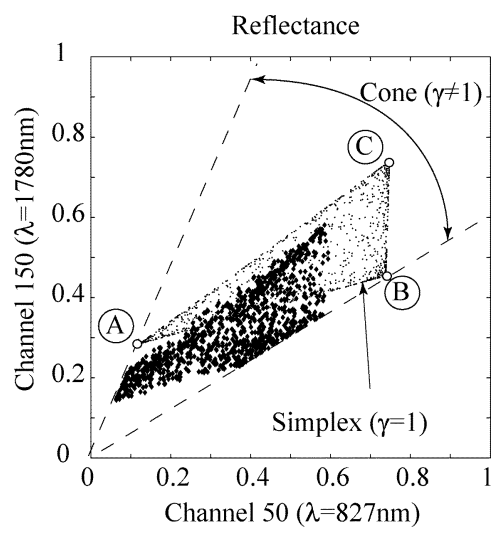

(a)

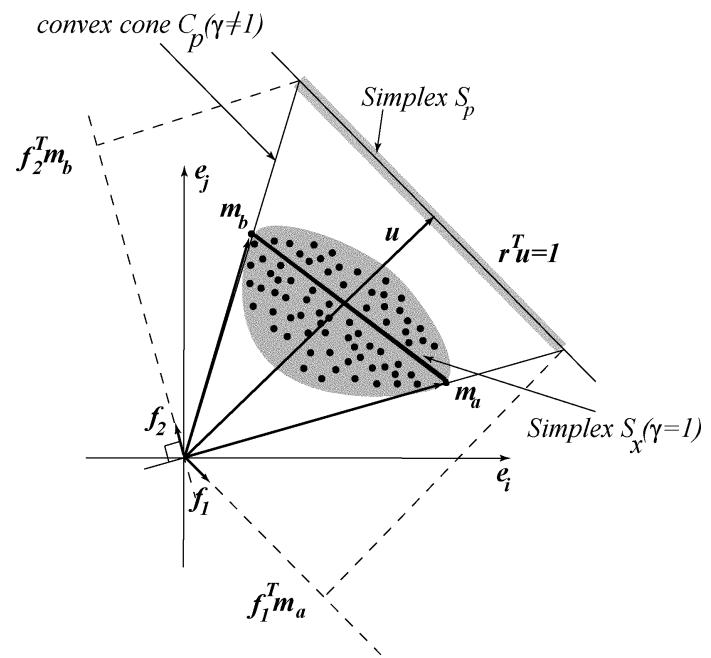

(b)

Fig. 1. (a) Two-dimensional scatterplot of mixtures of the three endmembers shown in Fig. 2. Circles denote pure materials. (b) Illustration of the VCA algorithm.

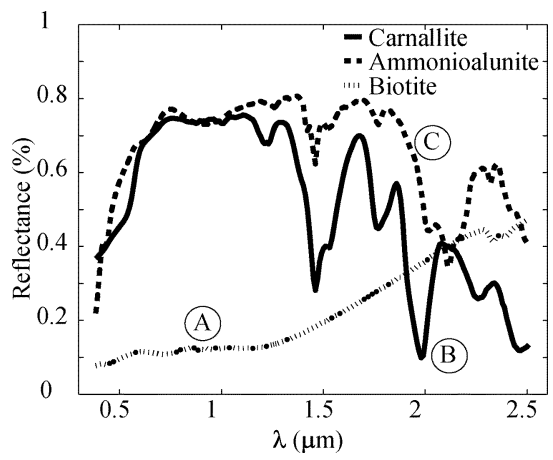

Fig. 2. Reflectances of carnallite, ammonioalunite, and biotite.

to significant savings in computational complexity and to SNR improvements.

Principal component analysis (PCA) [45], maximum-noise fraction (MNF) [46], and singular value decomposition (SVD) [47] are three well-known projection techniques widely used in remote sensing. PCA, also known as Karhunen-Loéve transform, seeks the projection that best represents data in a leastsquares sense; MNF seeks the projection that optimizes SNR; and SVD provides the projection that best represents data in the maximum-power sense. PCA and MNF are equal in the case

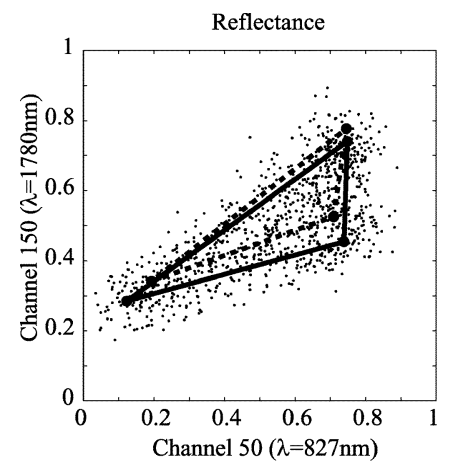

(a)

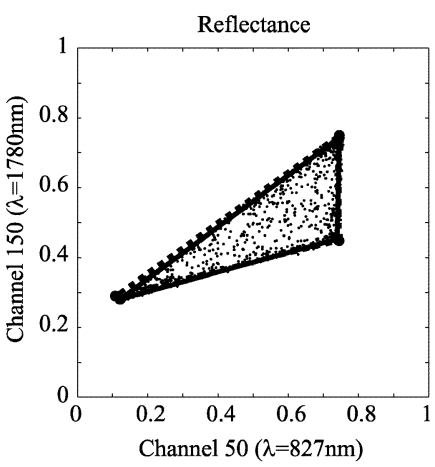

(b)
Fig. 3. Scatterplot (bands $\lambda=827 \mathrm{~nm}$ and $\lambda=1780 \mathrm{~nm}$ ) of the three endmembers mixture. (a) Unprojected data. (b) Projected data using SVD. Solid and dashed lines represent, respectively, simplexes computed from original and estimated endmembers (using VCA).

of white noise. SVD and PCA are also equal in the case of zero-mean data.

As discussed before, in the absence of noise, observed vectors $\mathbf{r}$ lie in a convex cone $C_{p}$ contained in a subspace $E_{p}$ of dimension $p$. The VCA algorithm starts by identifying $E_{p}$ by SVD and then projects points in $C_{p}$ onto a simplex $S_{p}$ by computing $\mathbf{y}=\mathbf{r} /\left(\mathbf{r}^{T} \mathbf{u}\right)$ [see Fig. 1(b)]. This simplex is contained in an affine set of dimension $p-1$. We note that the rational underlying the VCA algorithm is still valid if the observed dataset is projected onto any subspace $E_{d} \supset E_{p}$ of dimension $d$, for $p \leq d \leq L$, i.e., the projection of the cone $C_{p}$ onto $E_{d}$ followed by a projective projection is also a simplex with the same vertices. Of course, the SNR decreases as $d$ increases.

For illustration purposes, a simulated scene was generated according to (1). Three spectral signatures (A-biotite, B-carnallite, and C-ammonioalunite) were selected from the U.S. Geological Survey (USGS) digital spectral library [48] (see Fig. 2); the abundance fractions follow a Dirichlet distribution; parameter $\gamma$ is set to 1 ; and the noise is zero-mean white Gaussian with covariance matrix $\sigma^{2} \mathbf{I}$, where $\mathbf{I}$ is the identity matrix and $\sigma=0.045$ leading to a SNR $\equiv 10 \log _{10}\left(E\left[\mathbf{x}^{T} \mathbf{x}\right] / E\left[\mathbf{n}^{T} \mathbf{n}\right]\right)=20 \mathrm{~dB}$. Fig. 3(a) presents a scatterplot of the simulated spectral mixtures without projection (bands $\lambda=827 \mathrm{~nm}$ and $\lambda=1780 \mathrm{~nm}$ ). Two triangles are also plotted whose vertices represent the true endmembers (solid line) and the estimated endmembers (dashed line) by the VCA algorithm, respectively. Fig. 3(b) presents a scatterplot (same bands) of projected data onto the estimated affine set of dimension two inferred by SVD. Noise is clearly reduced, leading to a visible improvement on the VCA results.

As referred before, we apply the rescaling $\mathbf{r} /\left(\mathbf{r}^{T} \mathbf{u}\right)$ to get rid of the topographic modulation factor. As the SNR decreases, this rescaling amplifies noise, being preferable to identify directly the affine space of dimension $p-1$ by using only PCA. This phenomenon is illustrated in Fig. 4, where data clouds (noiseless and noisy) generated by two signatures are shown. Affines spaces $A_{p-1}$ and $A_{p-1}^{\prime}$ identified, respectively, by PCA of dimension $p-1$ and SVD of dimension $p$ followed by projective projection are schematized by straight lines. In the absence of noise, the direction of $\mathbf{m}_{a}$ is better identified by projective projection onto $A_{p-1}^{\prime}\left(\widehat{\mathbf{m}}_{a}\right.$ better than $\left.\widehat{\mathbf{m}}_{a}\right)$; in the presence of 
strong noise, the direction of $\mathbf{m}_{a}$ is better identified by orthogonal projection onto $A_{p-1}\left(\widehat{\hat{\mathbf{m}}}_{a}\right.$ better than $\left.\widehat{\mathbf{m}}_{a}\right)$. As a conclusion, when the SNR is higher than a given threshold $\mathrm{SNR}_{\mathrm{th}}$, data is projected onto $E_{p}$ followed by the rescaling $\mathbf{r} /\left(\mathbf{r}^{T} \mathbf{u}\right)$; otherwise data are projected onto $A_{p-1}$. Based on experimental results, we propose the threshold $\mathrm{SNR}_{\mathrm{th}}=15+10 \log _{10}(p) \mathrm{dB}$. Since for zero-mean white noise SNR $=E\left[\mathbf{x}^{T} \mathbf{x}\right] /\left(L \sigma^{2}\right)$, then we conclude that at $\mathrm{SNR}_{\mathrm{th}}, E\left[\mathbf{x}^{T} \mathbf{x}\right] /\left(p \sigma^{2}\right)=10^{1.5} L$, i.e., the $\mathrm{SNR}_{\mathrm{th}}$ corresponds to the fixed value $L \times 10^{1.5}$ of the SNR measured with respect to the signal subspace.

\section{B. VCA Algorithm}

The pseudocode for the VCA method is shown in Algorithm 1. Symbols $[\widehat{\mathbf{M}}]_{:, j}$ and $[\widehat{\mathbf{M}}]_{:, i: k}$ stand for the $j$ th column of $\widehat{\mathbf{M}}$ and for the $i$ th to $k$ th columns of $\widehat{\mathbf{M}}$, respectively. Symbol $\widehat{\mathbf{M}}$ stands for the estimated mixing matrix.

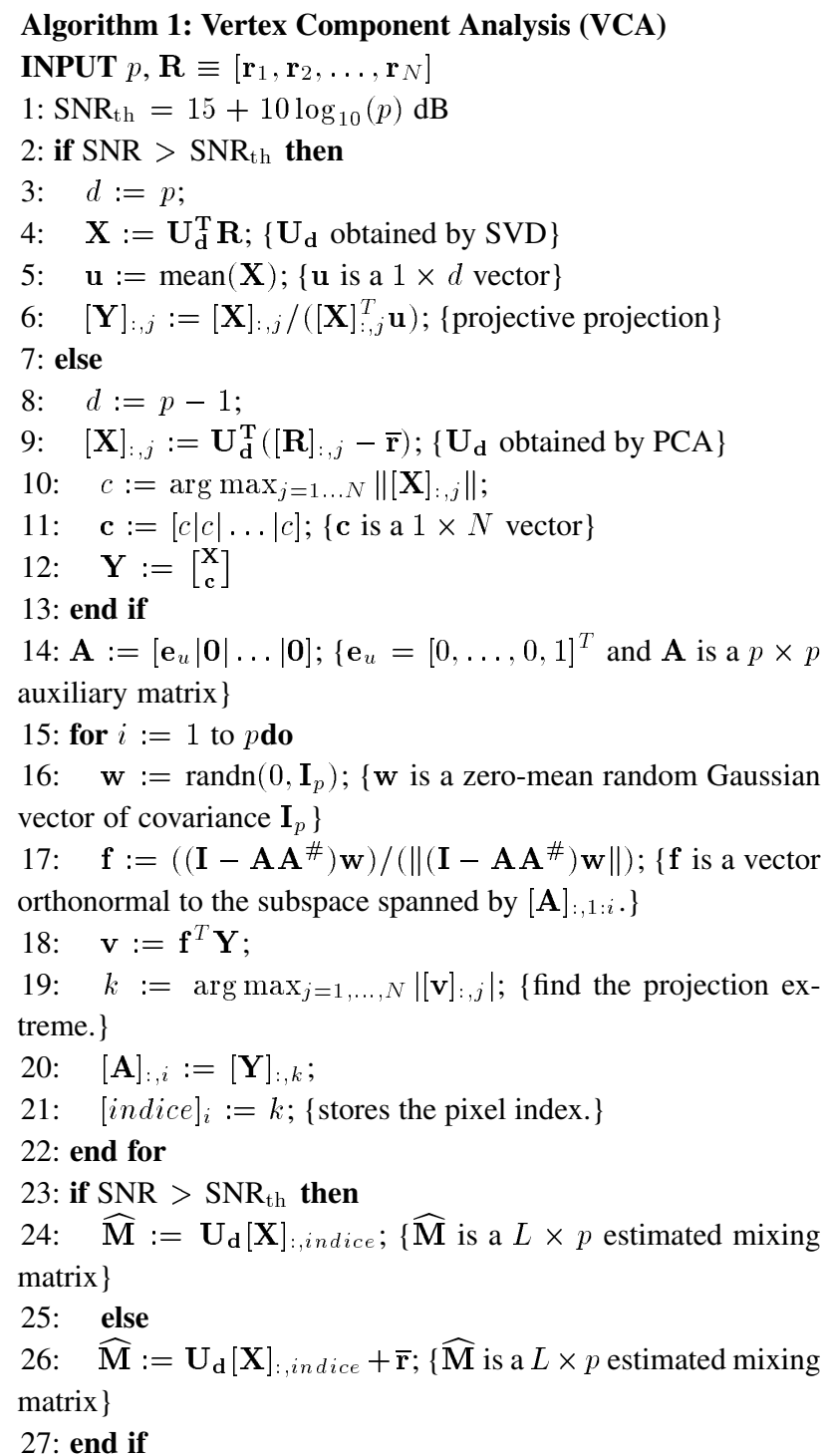

Step 2 tests if the SNR is higher than $\mathrm{SNR}_{\text {th }}$ in order to decide whether the data are to be projected onto a subspace of dimension $p$ or $p-1$. In the first case the projection matrix $\mathbf{U}_{\mathbf{d}}$ is

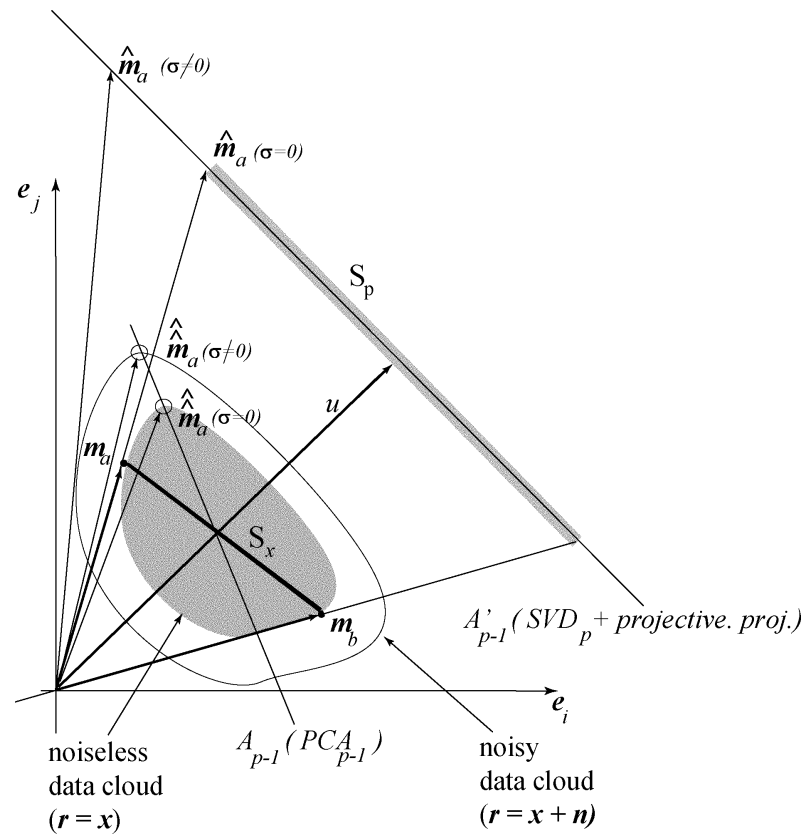

Fig. 4. Illustration of the noise effect on the dimensionality reduction.

obtained by SVD from $\mathbf{R R}^{T} / N$, where $\mathbf{R} \equiv\left[\mathbf{r}_{1}, \mathbf{r}_{2}, \ldots, \mathbf{r}_{N}\right]$ and $N$ is the number of pixels. In the second case the projection is obtained by PCA from $(\mathbf{R}-\overline{\mathbf{r}})(\mathbf{R}-\overline{\mathbf{r}})^{T} / N$, where $\overline{\mathbf{r}}$ is the sample mean of $[\mathbf{R}]_{:, i}$, for $i=1, \ldots, N$.

Steps 4 and 9 assure that the inner product between any vector $[\mathbf{X}]_{:, j}$ and vector $\mathbf{u}$ is nonnegative, a crucial condition for the VCA algorithm to work correctly. The chosen value of $c=$ $\arg \max _{j=1 \ldots N}\left\|[\mathbf{X}]_{:, j}\right\|$, assures that the colatitude angle between $\mathbf{u}$ and any vector $[\mathbf{X}]_{:, j}$ is between $0^{\circ}$ and $45^{\circ}$, then avoiding numerical errors which otherwise would occur for angles near $90^{\circ}$.

Step 14 initializes the auxiliary matrix $\mathbf{A}$, which stores the projection of the estimated endmembers signatures. Assume that there exists at least one pure pixel of each endmember in the input sample $\mathbf{R}$ [see Fig. 1(b)]. Each time the loop for is executed, a vector $\mathbf{f}$ orthonormal to the space spanned by the columns of the auxiliary matrix $\mathbf{A}$ is randomly generated and $\mathbf{y}$ is projected onto $\mathbf{f}$. Notation $(\cdot)^{\#}$ stands for the pseudoinverse matrix. Since we assume that pure endmembers occupy the vertices of a simplex, then $a \leq \mathbf{f}^{T}[\mathbf{Y}]_{:, i} \leq b$, for $i=1, \ldots, N$, where values $a$ and $b$ correspond to and only to pure pixels. We store the endmember signature corresponding to $\max (|a|,|b|)$. The next time loop for is executed, $\mathbf{f}$ is orthogonal to the space spanned by the signatures already determined. Since $\mathbf{f}$ is the projection of a zero-mean Gaussian independent random vector onto the orthogonal space spanned by the columns of $[\mathbf{A}]: 1: i$, then the probability of $\mathbf{f}$ being null is zero. Notice that the underling reason for generating a random vector is only to get a non null projection onto the orthogonal space generated by the columns of A. Fig. 1(b) shows the input samples and the chosen pixels, after the projection $\mathbf{v}=\mathbf{f}^{T} \mathbf{Y}$. Then a second vector $\mathbf{f}$ orthonormal to the endmember $a$ is generated and the second endmember is stored. Finally, steps 24 and 26 compute the columns of matrix $\widehat{\mathbf{M}}$, which contain the estimated endmembers signatures in the $L$-dimensional space. 


\section{EVAluAtion OF THE VCA AlgORIthM}

In this section, we compare VCA, PPI, and N-FINDR algorithms. N-FINDR and PPI were coded accordingly to [38] and [33], respectively. Regarding PPI, the number of skewers must be large [39], [40], [49]-[51]. Based on Monte Carlo runs, we concluded that the minimum number of skewers beyond which there is no unmixing improvements is about 1000. All experiments are based on simulated scenes from which we know the signature endmembers and their fractional abundances. Estimated endmembers are the columns of $\widehat{\mathbf{M}} \equiv\left[\widehat{\mathbf{m}}_{1}, \widehat{\mathbf{m}}_{2}, \ldots, \widehat{\mathbf{m}}_{p}\right]$. We also compare estimated abundance fractions given by $\widehat{\mathbf{S}}=\widehat{\mathbf{M}}^{\#}\left[\mathbf{r}_{1}, \mathbf{r}_{2}, \ldots, \mathbf{r}_{N}\right], \widehat{\mathbf{M}}^{\#}$ stands for pseudoinverse of $\widehat{\mathbf{M}}$ ) with the true abundance fractions.

To evaluate the performance of the three algorithms, we compute vectors of angles $\boldsymbol{\theta} \equiv\left[\theta_{1}, \theta_{2}, \ldots, \theta_{p}\right]^{T}$ and $\boldsymbol{\beta} \equiv\left[\beta_{1}, \beta_{2}, \ldots, \beta_{p}\right]^{T}$ with $^{1}$

$$
\begin{aligned}
\theta_{i} & \equiv\left(\arccos \frac{\left\langle\mathbf{m}_{i}, \widehat{\mathbf{m}}_{i}\right\rangle}{\left\|\mathbf{m}_{i}\right\|\left\|\widehat{\mathbf{m}}_{i}\right\|}\right) \\
\beta_{i} & \equiv\left(\arccos \frac{\left\langle[\mathbf{S}]_{i,:},[\widehat{\mathbf{S}}]_{i,:}\right\rangle}{\left\|[\mathbf{S}]_{i,:}\right\|\left\|[\widehat{\mathbf{S}}]_{i,:}\right\|}\right)
\end{aligned}
$$

where $\theta_{i}$ is the angle between vectors $\mathbf{m}_{i}$ and $\widehat{\mathbf{m}}_{i}$ (ith endmember signature estimate) and $\beta_{i}$ is the angle between vectors $[\mathbf{S}]_{i,:}$ and $[\widehat{\mathbf{S}}]_{i,:}$ (vectors of $\Re^{N}$ formed by the $i$ th lines of matrices $\widehat{\mathbf{S}}$ and $\mathbf{S} \equiv\left[\mathbf{s}_{1}, \mathbf{s}_{2}, \ldots, \mathbf{s}_{N}\right]$, respectively). The symmetric Kullback distance [52], a relative entropy-based distance, is another error measure used to compare similarity between signatures, namely under the name spectral information divergence (SID) [53]. SID is defined by

$$
\operatorname{SID}_{\mathbf{m}_{i}, \widehat{\mathbf{m}}_{i}} \equiv D\left(\mathbf{m}_{i} \mid \widehat{\mathbf{m}}_{i}\right)+D\left(\widehat{\mathbf{m}}_{i} \mid \mathbf{m}_{i}\right)
$$

where $D\left(\mathbf{m}_{i} \mid \widehat{\mathbf{m}}_{i}\right)$ is the relative entropy of $\mathbf{m}_{i}$ with respect to $\widehat{\mathbf{m}}_{i}$ given by

$$
D\left(\mathbf{m}_{i} \mid \widehat{\mathbf{m}}_{i}\right) \equiv \sum_{j=1}^{L} p_{j} \log \left(\frac{p_{j}}{q_{j}}\right)
$$

and $p_{j}=m_{i j} / \sum_{k=1}^{L} m_{i k}$ and $q_{j}=\widehat{m}_{i j} / \sum_{k=1}^{L} \widehat{m}_{i k}$.

Based on $\boldsymbol{\theta}, \boldsymbol{\beta}$, and $\boldsymbol{\phi} \equiv\left[\mathrm{SID}_{\mathbf{m}_{1}, \widehat{\mathbf{m}}_{1}}, \mathrm{SID}_{\mathbf{m}_{2}, \widehat{\mathbf{m}}_{2}}, \ldots\right.$, SID $\left.{ }_{\mathbf{m}_{p}, \widehat{\mathbf{m}}_{p}}\right]^{T}$, we estimate the following rms error distances:

$$
\begin{aligned}
\epsilon_{\theta} & =\left(\frac{1}{p} E\left[\|\boldsymbol{\theta}\|_{2}^{2}\right]\right)^{1 / 2} \\
\epsilon_{\phi} & =\left(\frac{1}{p} E\left[\|\boldsymbol{\phi}\|_{2}^{2}\right]\right)^{1 / 2} \\
\epsilon_{\beta} & =\left(\frac{1}{p} E\left[\|\boldsymbol{\beta}\|_{2}^{2}\right]\right)^{1 / 2}
\end{aligned}
$$

where $E[\cdot]$ denotes the expectation operator. The first two quantities measure distances between $\widehat{\mathbf{m}}_{i}$ and $\mathbf{m}_{i}$, for $i=1, \ldots, p$; the third is similar to the first, but for the estimated abundance

\footnotetext{
${ }^{1}$ Notation $\langle\mathbf{x}, \mathbf{y}\rangle$ stands for the inner product $\mathbf{x}^{T} \mathbf{y}$.
}

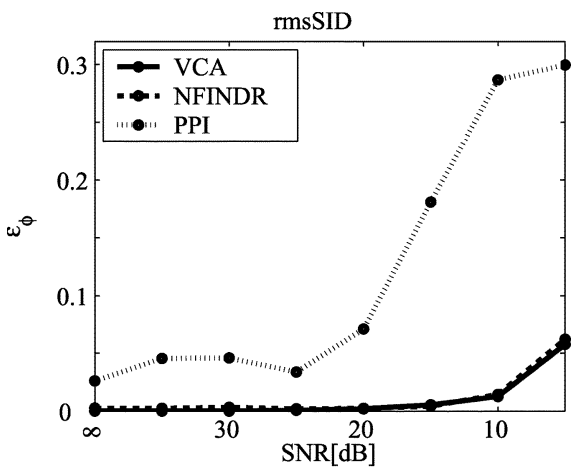

(a)

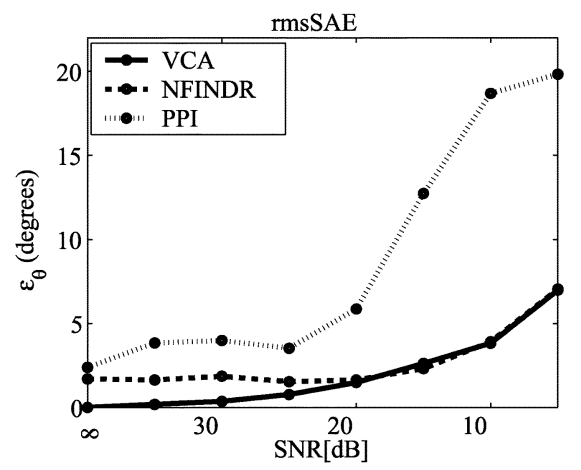

(b)

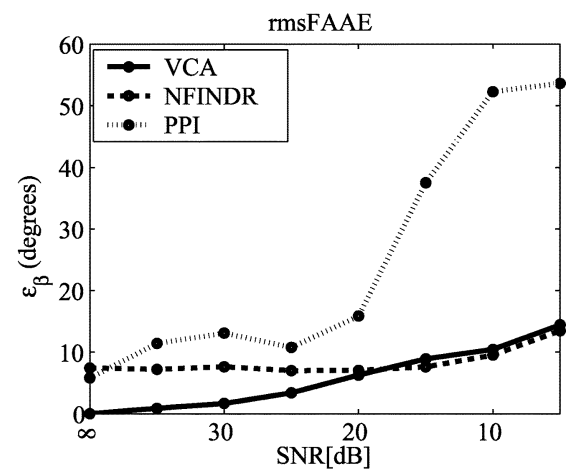

(c)

Fig. 5. First scenario $\left(N=1000, p=3, L=224, \mu_{1}=\mu_{2}=\mu_{3}=1 / 3\right.$, $\beta_{1}=20, \beta_{2}=1$ ). (a) rmsSID as function of SNR. (b) rmsSAE as function of SNR. (c) rmsFAAE as function of SNRs.

fractions. Herein we name $\epsilon_{\theta}, \epsilon_{\phi}$, and $\epsilon_{\beta}$ as rmsSAE, rmsSID, and rmsFAAE, respectively (SAE stands for signature angle error and FAAE stands for fractional abundance angle error). Mean values in (6)-(8) are approximated by sample means based on 100 Monte Carlo runs.

In all experiments, the spectral signatures are selected from the USGS digital spectral library [48]. Fig. 2 shows three of these endmember signatures. Abundance fractions are generated according to a Dirichlet distribution given by

$$
\begin{aligned}
& p\left(\alpha_{1}, \alpha_{2}, \ldots, \alpha_{p}\right)=\frac{\Gamma\left(\mu_{1}+\mu_{2}+\ldots+\mu_{p}\right)}{\Gamma\left(\mu_{1}\right) \Gamma\left(\mu_{2}\right) \ldots \Gamma\left(\mu_{p}\right)} \\
& \times \alpha_{1}^{\mu_{1}-1} \alpha_{2}^{\mu_{2}-1} \ldots \alpha_{p}^{\mu_{p}-1}
\end{aligned}
$$

where $0 \leq \alpha_{i} \leq 1, \sum_{i=1}^{p} \alpha_{i}=1, E\left[\alpha_{i}\right]=\mu_{i} / \sum_{k=1}^{p} \mu_{k}$ is the expected value of the $i$ th endmember fraction, and $\Gamma(\cdot)$ denotes the Gamma function. Parameter $\gamma$ is $\operatorname{Beta}\left(\beta_{1}, \beta_{2}\right)$ distributed, 


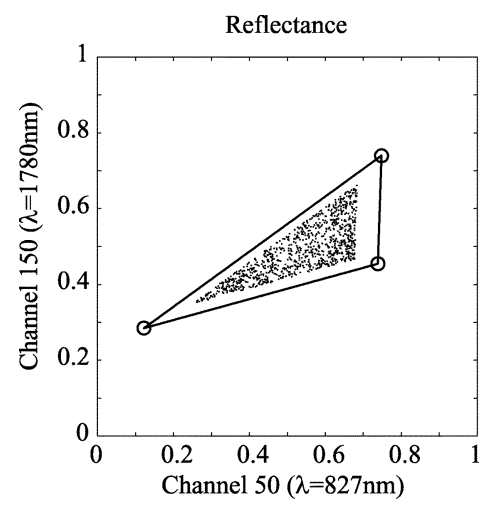

Fig. 6. Illustration of the absence of pure pixels $(N=1000, p=3, L=$ $224, \mu_{1}=\mu_{2}=\mu_{3}=1 / 3, \gamma=1$ ). Scatterplot (bands $\lambda=827 \mathrm{~nm}$ and $\lambda=1780 \mathrm{~nm}$ ), with fractional abundance smaller than 0.2 rejected.

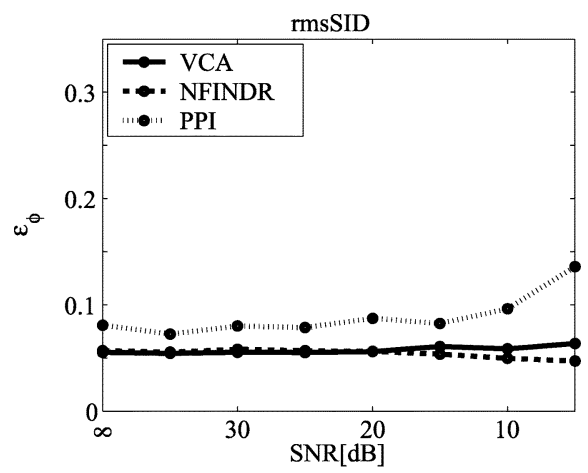

(a)

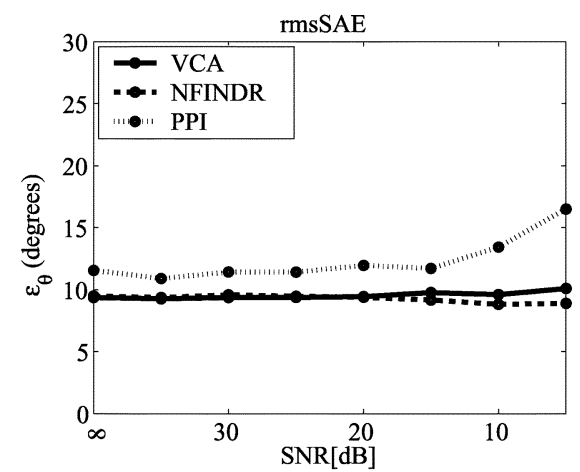

(b)

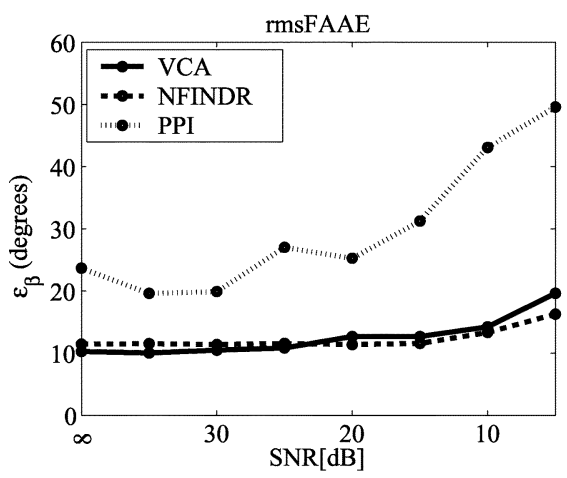

(c)

Fig. 7. Robustness to the absence of pure pixels $(N=1000, p=3, L=$ $224, \mu_{1}=\mu_{2}=\mu_{3}=1 / 3, \beta_{1}=20, \beta_{2}=1$ ). (a) rmsSID as function of SNR. (b) rmsSAE as function of SNR. (c) rmsFAAE as function of SNR.

i.e., $p(\gamma)=\left(\Gamma\left(\beta_{1}+\beta_{2}\right) / \Gamma\left(\beta_{1}\right) \Gamma\left(\beta_{2}\right)\right) \gamma^{\beta_{1}-1}(\gamma-1)^{\beta_{2}-1}$, which is also a Dirichlet distribution. The Dirichlet density, besides

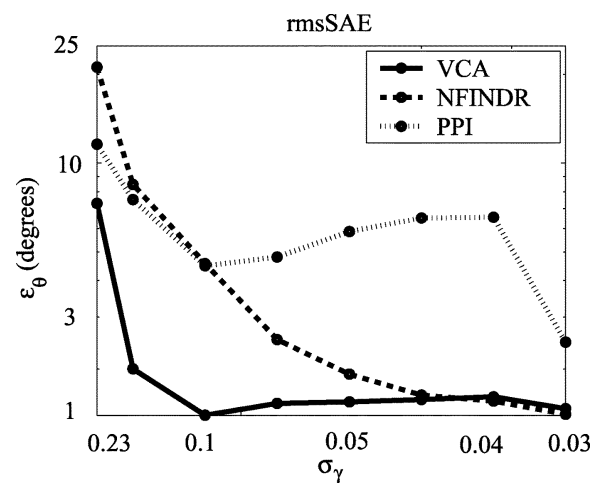

Fig. 8. Robustness to the topographic modulation $(N=1000, p=3, L=$ $224, \mu_{1}=\mu_{2}=\mu_{3}=1 / 3$, SNR $=20 \mathrm{~dB}, \beta_{2}=1$ ), rmsSEA as function of the $\sigma_{\gamma}^{2}$ (variance of $\gamma$ ).

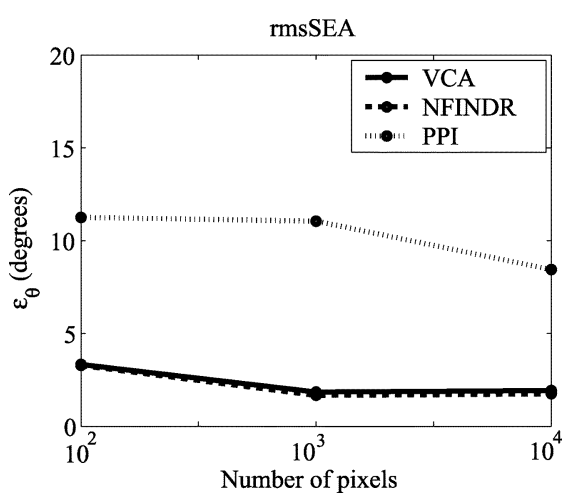

Fig. 9. The rmsSEA as function of the number of pixels in a scene ( $p=6$, $\left.L=224, \mu_{1}=\mu_{2}=\mu_{3}=1 / 3, \mathrm{SNR}=20 \mathrm{~dB}, \beta_{2}=20, \beta_{2}=1\right)$.

enforcing positivity and full additivity constraints, displays a wide range of shapes, depending on the parameters $\mu_{1}, \ldots, \mu_{i}$. This flexibility underlies its choice in our simulations.

The results next presented are organized into five experiments: in the first experiment, the algorithms are evaluated with respect to the SNR and to the absence of pure pixels. As mentioned before, we define

$$
\mathrm{SNR} \equiv 10 \log _{10} \frac{E\left[\mathbf{x}^{T} \mathbf{x}\right]}{E\left[\mathbf{n}^{T} \mathbf{n}\right]}
$$

In the case of zero-mean noise with covariance $\sigma^{2} \mathbf{I}$ and Dirichlet abundance fractions, one obtains

$$
\mathrm{SNR}=10 \log _{10} \frac{\operatorname{tr}\left[\mathbf{M} \mathbf{R}_{\mathbf{s}} \mathbf{M}^{T}\right]}{L \sigma^{2}}
$$

where

$$
\mathbf{R}_{\mathrm{s}} \equiv \sigma_{\gamma}^{2} E\left[\boldsymbol{\alpha} \boldsymbol{\alpha}^{T}\right]=\sigma_{\gamma}^{2} \frac{\boldsymbol{\mu} \boldsymbol{\mu}^{T}+\operatorname{diag}(\boldsymbol{\mu})}{\left(\sum_{i=1}^{p} \mu_{i}\right)\left(1+\sum_{i=1}^{p} \mu_{i}\right)}
$$

$\mu=\left[\mu_{1} \ldots \mu_{p}\right]^{T}$, and $\sigma_{\gamma}^{2}$ is the variance of parameter $\gamma$. For example, assuming abundance fractions equaly distributed, we have, after some algebra, $\mathrm{SNR} \simeq 10 \log _{10} \sigma_{\gamma}^{2} \sum_{i=1}^{p}\left(\sum_{j=1}^{p} m_{i j}^{2} / p\right) /\left(L \sigma^{2}\right)$ for $\mu p \ll 1$ and $\mathrm{SNR} \simeq 10 \log _{10} \sigma_{\gamma}^{2}\left(\sum_{i=1}^{p}\left(\sum_{j=1}^{p} m_{i j}\right)^{2} / p^{2}\right) /\left(L \sigma^{2}\right)$ for $\mu p \gg 1$. 
In the second experiment, the performance is measured as function of the parameter $\gamma$, which models fluctuations on the illumination due to the surface topography. In the third experiment, the number of pixels of the scene varies, in order to illustrate the algorithm performance with the size of the covered area: as the number of pixels increases, the likelihood of having pure pixels also increases, improving the performance of the unmixing algorithms; in the fourth experiment, the algorithms are evaluated as function of the number of endmembers present in the scene; finally, in the fifth experiment, the number of floating-point operations (flops) is measured, in order to compare the computational complexity of VCA, N-FINDR, and PPI algorithms.

In the first experiment, the hyperspectral scene has 1000 pixels and the abundance fractions are Dirichlet distributed with $\mu_{i}=1 / 3$, for $i=1,2,3$; parameter $\gamma$ is Beta distributed with $\beta_{1}=20$ and $\beta_{2}=1$ implying $E[\gamma]=0.952$ and $\sigma_{\gamma}=0.05$.

Fig. 5 shows performance results as function of the SNR. As expected, the presence of noise degrades the performance of all algorithms. In terms of rmsSID, VCA, and N-FINDR algorithms have identical performances, whereas PPI displays the worst result. In terms of rmsSAE and rmsFAAE [Fig. 5(b) and (c)], we can see that when SNR is less than $20 \mathrm{~dB}$ VCA algorithm exhibits the best performance. Note that for noiseless scenes, only the VCA algorithm has zero rmsSAE.

Fig. 7 shows performance results as function of the SNR in the absence of pure pixels. Spectral data without pure pixels was obtained by rejecting pixels with any fractional abundance smaller than 0.2. Fig. 6 shows the obtained scatter plot. VCA and N-FINDR display similar results, being both better than PPI. Notice that the performance is almost independent of the SNR and is uniformly worse than that displayed with pure pixels and $\mathrm{SNR}=5 \mathrm{~dB}$ in the first experiment. We conclude that this family of algorithms is more affected by the lack of pure pixels than by low SNR.

For economy of space and also because rmsSID, rmsSAE, and rmsFAAE disclose similar pattern of behavior, we only present the rmsSAE in the remaining experiments.

In the second experiment, abundance fractions are generated as in the first one, SNR is set to $20 \mathrm{~dB}$, and parameter $\gamma$ is Beta distributed with $\beta_{2}=1$ and $\beta_{1}$ in the interval $[2,28]$. This corresponds to vary $E[\gamma]$ from 0.66 to 0.96 and $\sigma_{\gamma}$ from 0.23 to 0.03 . By varying parameter $\beta_{1}$, the severity of topographic modulation is also varied. Fig. 8 illustrates the effect of topographic modulation on the performance of the three algorithms. When $\beta_{1}$ grows ( $\sigma_{\gamma}$ gets smaller) the performance improves. This is expected, since the simplex identification is more accurate when the topographic modulation is smaller. PPI algorithm displays the worst performance for $\sigma_{\gamma}<0.1$. VCA and N-FINDR algorithms have identical performances when $\beta_{1}$ takes higher values $\left(\sigma_{\gamma}<0.045\right)$; otherwise the VCA algorithm has the best performance. VCA is more robust to topographic modulation, since it seeks for the extreme projections of the simplex, whereas $\mathrm{N}$-FINDR seeks for the maximum volume, which is more sensitive to fluctuations on $\gamma$.

In the third experiment, the number of pixels is varied, the abundance fractions are generated as in the first one, and SNR = 20 dB. Fig. 9 shows that VCA and N-FINDR exhibit identical

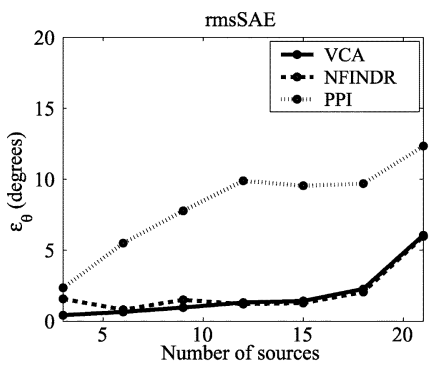

(a)

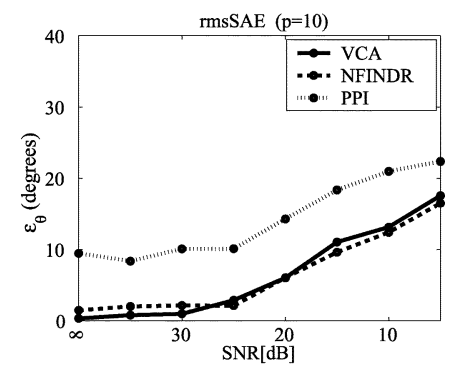

(b)
Fig. 10. Impact of the number of endmembers $\left(N=1000, L=224, \mu_{1}=\right.$ $\mu_{2}=\mu_{3}=1 / 3, \mathrm{SNR}=30 \mathrm{~dB}, \beta_{2}=20, \beta_{2}=1$ ). (a) rmsSEA as function of the number of endmembers. (b) rmsSEA function of the SNR with $p=10$.

TABLE I

COMPUTATIONAL COMPLEXITY OF VCA, N-FINDR, AND PPI ALGORITHMS

\begin{tabular}{l|l}
\hline Algorithm & Complexity (flops) \\
\hline VCA & $2 p^{2} N$ \\
N-FINDR & $p^{\eta+1} N$ \\
PPI & $2 p s N$ \\
\hline
\end{tabular}

results, whereas, the PPI algorithm displays the worst result. Note that the behavior of the three algorithms is quasi independent of the number of pixels.

In the fourth experiment, we vary the number of signatures from $p=3$ to $p=21$, the scene has 1000 pixels, and $\mathrm{SNR}=30 \mathrm{~dB}$. Fig. 10(a) shows that VCA and N-FINDR performances are comparable, while PPI displays the worst result. The rmsSAE increase slightly as the number of endmembers present in the scene increases. It is also plotted the rmsSAE as function of the SNR with $p=10$ [see Fig. 10(b)]. Compared with Fig. 5(b), we conclude that when the number of endmembers increases the performance of the algorithms slightly decreases.

In the fifth and last experiment, the number of flops is measured, in order to compare the computational complexity of VCA, PPI, and N-FINDR algorithms. Herein, we use the scenarios of the second and third experiments. Table I presents approximated expressions for the number of flops used by each algorithm. These expressions do not account for the computational complexities involved in the computations of the sample covariance $(\mathbf{R}-\overline{\mathbf{r}})(\mathbf{R}-\overline{\mathbf{r}})^{T} / N$ nor in the eigendecomposition. The reason is that these operations, compared with the VCA, PPI, and N-FINDR algorithms, have a negligible computational cost, since the following.

- The computation of $(\mathbf{R}-\overline{\mathbf{r}})(\mathbf{R}-\overline{\mathbf{r}})^{T} / N$ has a complexity of $2 N L^{2}$ flops. However, in practice one does not need to use the complete set of $N$ hyperspectral vectors. If the scene is noiseless, only $p-1$ linearly independent vectors would be enough to infer the exact subspace $E_{p-1}$. In the presence of noise, however, a larger set should be used. For example, in a $1000 \times 1000$ hyperspectral image, we found out that only 1000 samples randomly sampled are enough to find a very good estimate of $E_{p-1}$. Even a sample size of 100 leads to good results on this respect.

- Concerning the eigendecomposition of $(\mathbf{R}-\overline{\mathbf{r}})(\mathbf{R}-$ $\overline{\mathbf{r}})^{T} / N$ (or the SVD of $\mathbf{R R}^{T} / N$ ), we only need to compute $p-1$ (or $p$ ) eigenvectors corresponding to the largest 


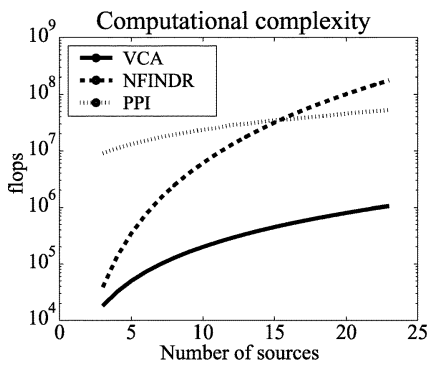

(a)

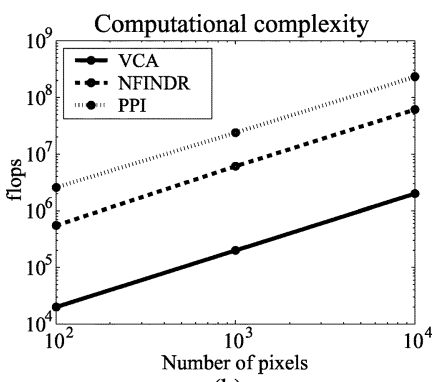

(b)
Fig. 11. Computational complexity measured in number of flops.

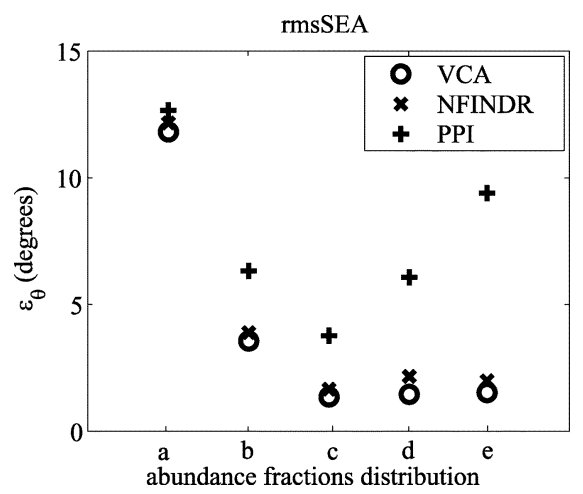

Fig. 12. The rmsSEA as function of different abundance fractions distributions $\left(N=1000, p=3, L=224, \mathrm{SNR}=20 \mathrm{~dB}, \beta_{2}=20, \beta_{2}=1\right)$. From left to right: (a) $\beta=1 / 2$, (b) $\beta=1$, (c) $\beta=2$ ), (d) $\beta=3$, (e) Dirichlet density $\left(\mu_{1}=\mu_{2}=\mu_{3}=1 / 3\right)$.

$p-1$ eigenvalues (or $p$ single values). For these partial eigendecomposition, we have used the PCA algorithm [45] (or SVD analysis [47]) whose complexity is negligible compared with the remaining operations.

The VCA algorithm projects all data ( $N$ vectors of size $p$ ) onto $p$ orthogonal directions. N-FINDR computes $p N$ times the determinant of a $p \times p$ matrix, whose complexity is $p^{\eta}$, with $2.3<\eta<2.9$ [54]. Assuming that $N \gg p>2$, VCA complexity is lower than that of N-FINDR. Concerning PPI, given that the number of skewers $(s)$ is much higher than the usual number of endmembers, the PPI complexity is much higher than that of VCA. We conclude, then, that the VCA algorithm has always the lowest complexity.

Fig. 11 plots the flops for the three algorithms after data projection. In Fig. 11(a), the abscissa is the number of endmembers in the scene, whereas in Fig. 11(b), the abscissa is the number of pixels. Note that for five endmembers, VCA computational complexity is one order of magnitude lower than that of the N-FINDR algorithm. When the number of endmembers is higher than 15 , the VCA computational complexity is at least two orders of magnitude lower than PPI and N-FINDR algorithms.

The results presented in this section were based on abundance fractions with symmetric Dirichlet distribution. The same pattern of behavior was, however, found for any other abundance fraction distribution tested. Fig. 12 shows the results for abundance fractions $\alpha_{i}=x_{i}^{\beta} / \sum_{i}^{p} x_{i}^{\beta}$ for $i=1, \ldots, p$, with $x_{i}$ uniformly distributed in $[0,1]$ and $\beta=1 / 2$ (a), $\beta=1$ (b), $\beta=2$ (c), and $\beta=3$ (d). Dirichlet density with $\mu_{1}=\mu_{2}=\mu_{3}=$

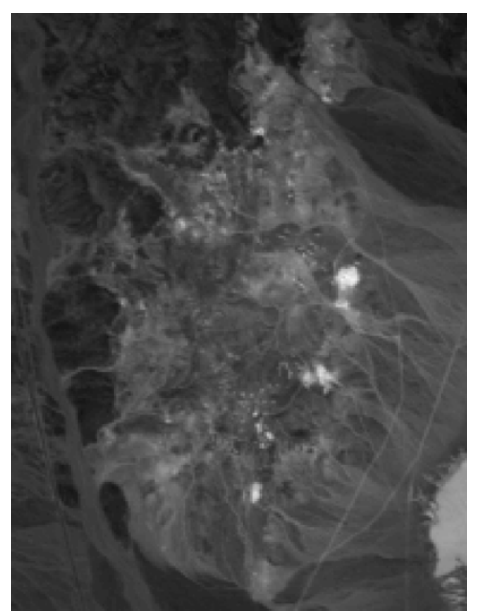

Fig. 13. Band $30(\lambda=667.3 \mathrm{~nm})$ of a subimage of the AVIRIS Cuprite Nevada dataset.

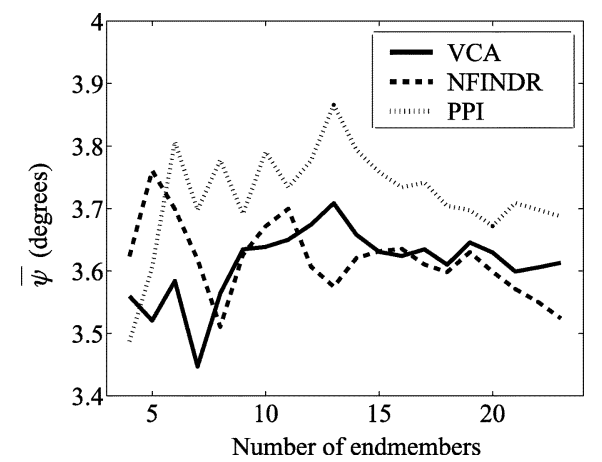

Fig. 14. $\bar{\psi}$ as function of the number of endmembers.

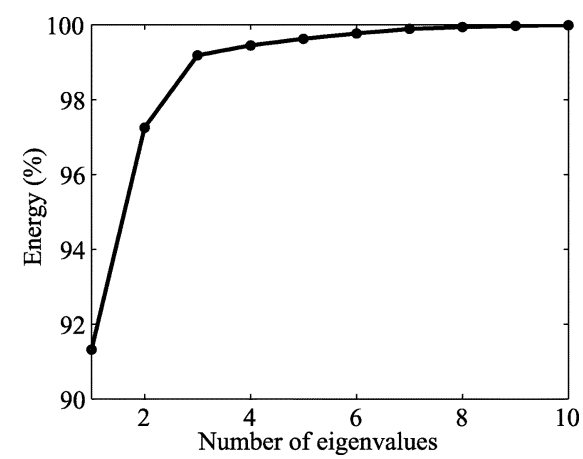

Fig. 15. Percentage of signal energy as a function of the number of eigenvalues.

$1 / 3$ is included (e) for comparison purposes. The VCA algorithm display always the lowest rmsSEA. The performance increase for all algorithms as $\beta$ increases, because the likelihood of having pure pixels increases with $\beta$.

In the introduction, besides PPI and N-FINDR algorithms, we have also mentioned ORASIS. Nevertheless, no comparison whatsoever was made with this method. The reason is that there is no ORASIS implementation details published in open the literature. We can, however, make a few considerations based on the results recently published in [51]. This work compares, among others, PPI, N-FINDR, and ORASIS algorithms. Although the relative performance of the three algorithms varies, depending on SNR, number of endmembers, spectral signatures, type of atmospheric correction, etc., both PPI and 


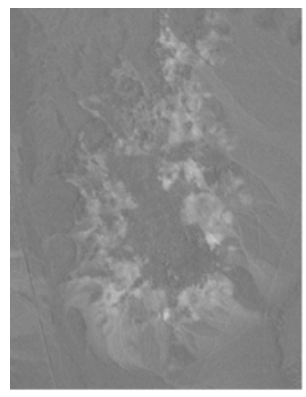

(a)

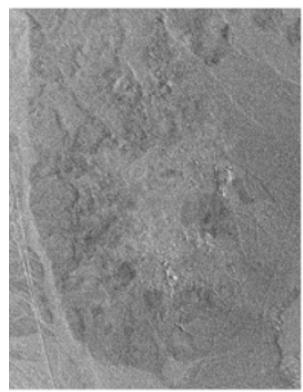

(e)

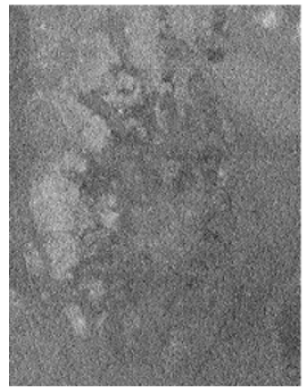

(i)

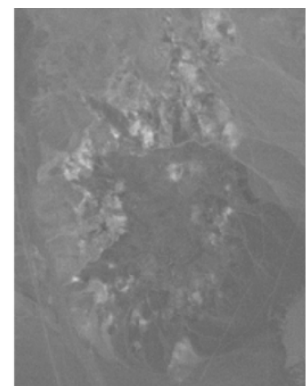

(b)

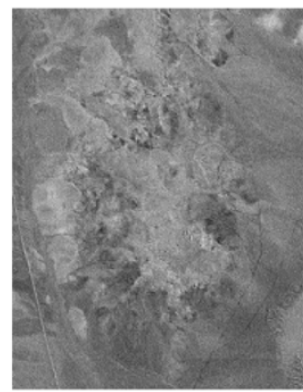

(f)

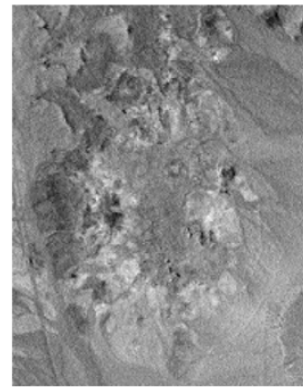

(j)

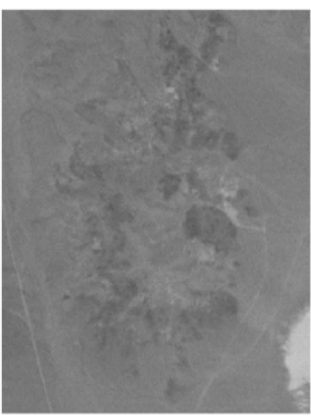

(m)

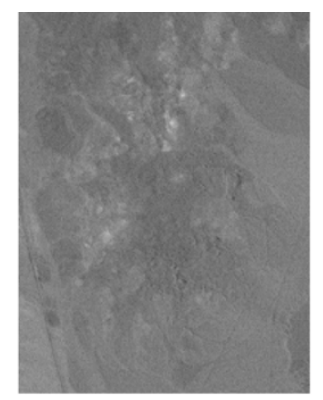

(c)

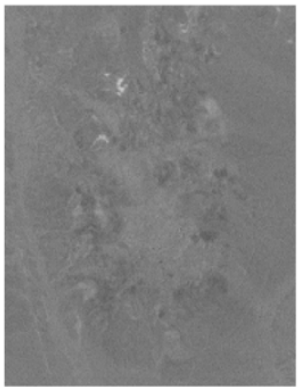

(g)

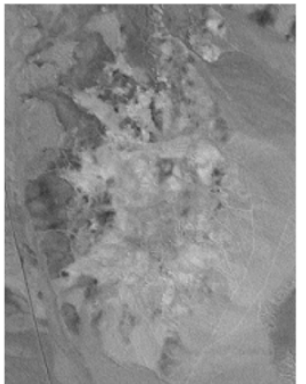

(k)

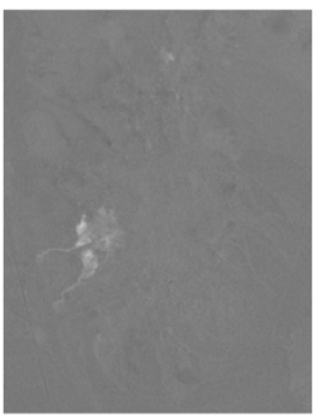

(n)

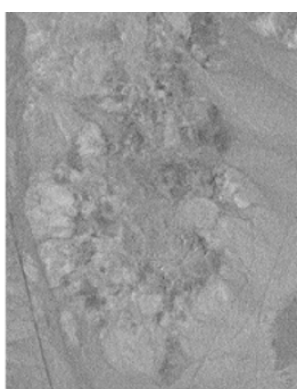

(d)

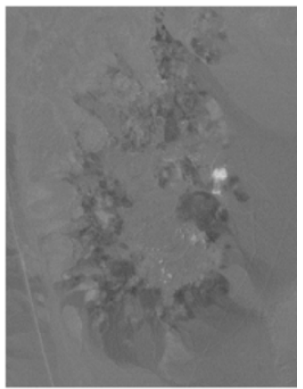

(h)

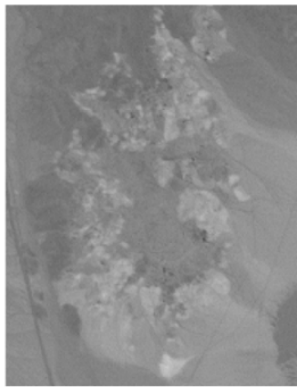

(l)

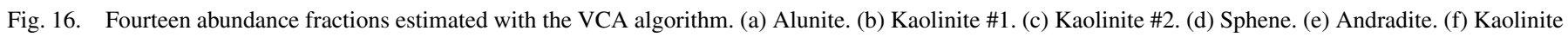
\#3. (g) Nontronite. (h) Muscovite. (i) Pyrope \#1. (j) Pyrope \#2. (k) Kaolinite \#4. (1) Dumortierite. (m) Montmorillonite. (n) Buddingtonite.

N-FINDR generally perform better than ORASIS when SNR is low. Since in all comparisons herein conducted, VCA performs better than or equal to PPI and N-FINDR, we expect that the proposed method performs better than or equal to ORASIS when low SNR dominates the data, although further experiments would be required to demonstrate the above remark.

\section{EVAluATION WITH EXPERIMENTAL DATA}

In this section, we apply the VCA algorithm to real hyperspectral data collected by the AVIRIS [4] sensor over Cuprite,
NV. Cuprite is a mining area in southern Nevada with mineral and little vegetation [55], located approximately $200 \mathrm{~km}$ northwest of Las Vegas. The test site is a relatively undisturbed acid-sulphate hydrothermal system near U.S. Highway 95. The geology and alteration were previously mapped in detail [56], [57]. A geologic summary and a mineral map can be found in [55] and [58]. This site has been extensively used for remote sensing experiments over the past years [59], [60].

Our study is based on a subimage $(250 \times 190$ pixels and 224 bands) of a dataset acquired on the AVIRIS flight June 19, 1997 (see Fig. 13). In order to compare results with a signature library, we process the reflectance image after atmospheric correction. 


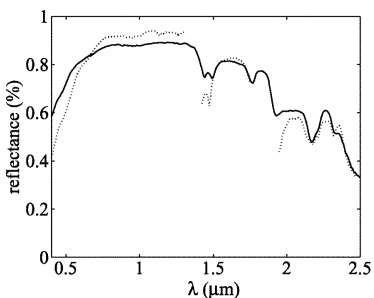

(a)

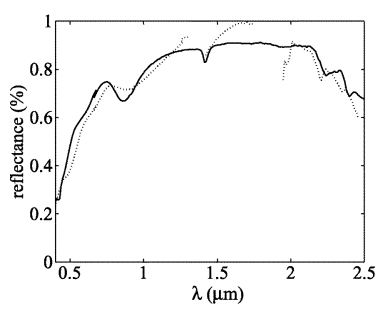

(e)

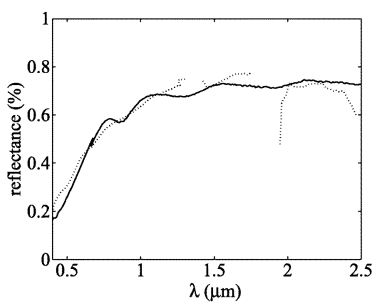

(i)

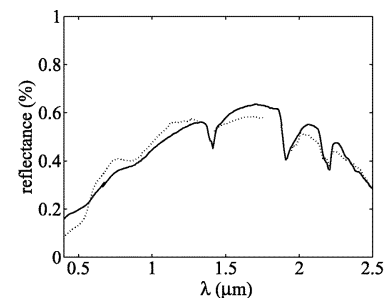

(b)

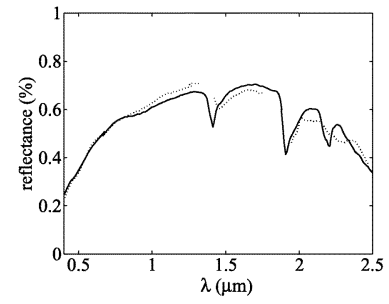

(f)

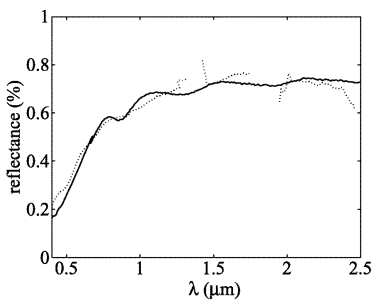

(j)

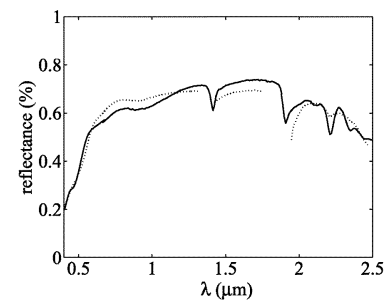

(m)

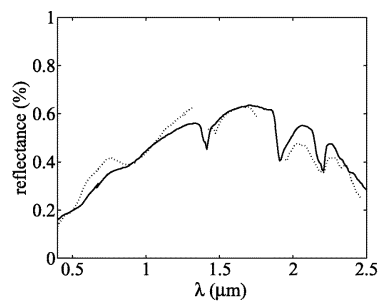

(c)

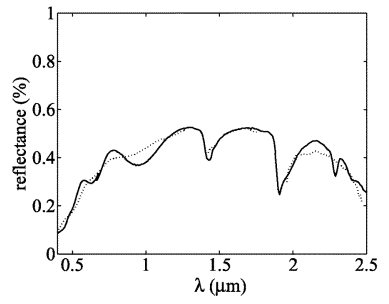

(g)

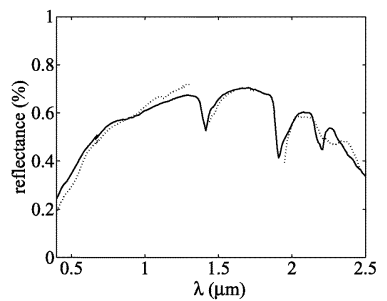

(k)

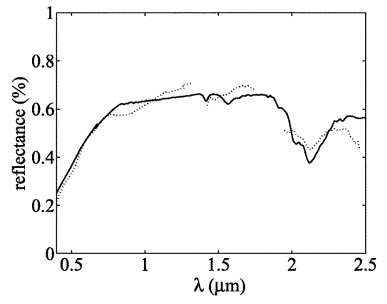

(n)

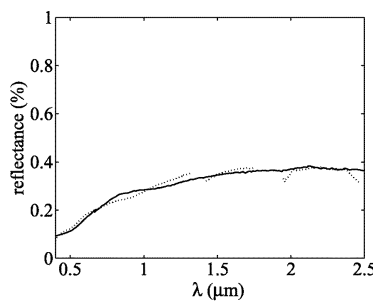

(d)

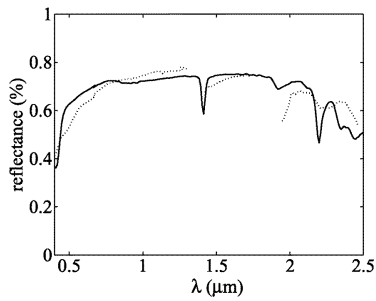

(h)

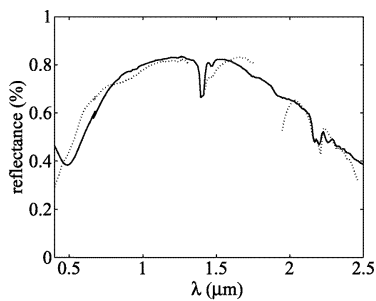

(1)

Fig. 17. Comparison of (dotted line) the extracted signatures with (solid line) the USGS spectral library. (a) Alunite. (b) Kaolinite \#1. (c) Kaolinite \#2. (d) Sphene. (e) Andradite. (f) Kaolinite \#3. (g) Nontronite. (h) Muscovite. (i) Pyrope \#1. (j) Pyrope \#2. (k) Kaolinite \#4. (l) Dumortierite. (m) Montmorillonite. (n) Buddingtonite.

To determine the type of projection applied by VCA, we compute

$$
\mathrm{SNR} \simeq 10 \log _{10} \frac{P_{R_{p}}-\left(\frac{p}{L}\right) P_{R}}{P_{R}-P_{R_{p}}}
$$

where $P_{R} \equiv E\left[\mathbf{r}^{T} \mathbf{r}\right]$ and $P_{R_{p}}=E\left[\mathbf{r}^{T} \mathbf{U}_{d} \mathbf{U}_{d}^{T} \mathbf{r}\right]$ in the case of SVD and $P_{R_{p}} \equiv E\left[\mathbf{r}^{T} \mathbf{U}_{d} \mathbf{U}_{d}^{T} \mathbf{r}\right]+\overline{\mathbf{r}}^{T} \overline{\mathbf{r}}$ in the case of PCA.

In order to compare the performance of the three algorithms when applied to real data, we compute

$$
\bar{\psi} \equiv \frac{\sum_{i} \psi_{i} E_{i}}{\sum_{i} E_{i}}
$$

where $\psi_{i}$ is the angle between the $i$ th estimated signature, $\widehat{\mathbf{m}}_{i}$, and the nearest laboratory spectra, and $E_{i} \equiv(1 / N) \sum_{j=1}^{N} \mid<$ $\mathbf{y}_{j}^{T}, \widehat{\mathbf{m}}_{i} /\left\|\widehat{\mathbf{m}}_{i}\right\|>\left.\right|^{2}$ is the sample mean energy of the hyperspectral image projected onto $\widehat{\mathbf{m}}_{i}$. Therefore, $\bar{\psi}$ is a mean angle error weighted by the energy on the direction $\widehat{\mathbf{m}}_{i}$. Fig. 14 presents the performance of the three algorithms, as function of the number of endmembers. VCA and N-FINDR performances are comparable and better than PPI one, when the number of endmembers varies from 4 to 23 .

In order to estimate the number of endmembers present in the processed area, we resort to the virtual dimensionality (VD), recently proposed in [61]. The VD is defined as the minimum number of spectrally distinct signal sources that characterize the hyperspectral data, from the target detection and classification point of view. Harsanyi et al. developed a Neyman-Pearson detection theory-based thresholding method (HFC) to determine the VD of hyperspectral imagery, where eigenvalues are used to measure signal energies in a detection model [62]. A modified version of this method called noise-whitened HFC (NWHFC) includes a noise-whitening process as preprocessing step to remove the second-order statistical correlation [17]. In the NWHFC method a noise estimation is required.

By specifying a false-alarm probability $P_{f}$, a Neyman-Pearson detector is derived to determine whether or not a distinct signature is present in each of spectral bands. How many times the Neyman-Pearson detector fails 
TABLE II

SPECTRAL ANGLE Distance BETWEEN EXTRACTED ENDMEMBERS AND LABORATORY REFLECTANCES FOR VCA, N-FINDR, AND PPI ALGORITHMS

\begin{tabular}{l|r|r|r}
\hline & VCA & N-FINDR & PPI \\
\hline Substance & $\bar{\psi}=3.65$ & $\bar{\psi}=3.62$ & $\bar{\psi}=3.79$ \\
\hline Alunite & 4.1 & 4.0 & 4.2 \\
Kaolinite \#1 & 4.8 & 4.8 & 4.8 \\
Kaolinite \#2 & 5.5 & 5.2 & 5.2 \\
Sphene & 3.1 & Andalusite $(2.9)$ & 3.1 \\
Andradite & 3.5 & 4.4 & Hematite (3.7) \\
Kaolinite \#3 & 2.7 & 2.6 & 2.6 \\
Nontronite & 3.9 & 4.0 & 3.8 \\
Muscovite & 4.1 & Erionite $(5.2)$ & 6.1 \\
Pyrope \#1 & 3.7 & Barite (4.0) & Barite (4.0) \\
Pyrope \#2 & 3.1 & 3.4 & 3.2 \\
Kaolinite \#4 & 3.1 & Montmoril.(3.5) & Montmoril.(4.2) \\
Dumortierite & 3.8 & 4.1 & 5.6 \\
Montmorillonite & 3.0 & 3.1 & 2.9 \\
Buddingtonite & 3.8 & 4.3 & 3.9 \\
\hline
\end{tabular}

the test is exactly the number of endmembers assumed to be present in the data.

By inspection of the eigenvalues of the sample covariance matrix and of the sample correlation matrix of the subimage, the VD was estimated by the NWHFC-based eigenthresholding method using the Neyman-Pearson test with the false-alarm probability set to $P_{f}=10^{-5}$. This method leads to an estimation of the number of endmembers equal to $p=14$.

Fig. 15 presents the cumulative signal energy as function of the number of eigenvalues. We can see that the signal energy contained in the first eight eigenvalues is higher than $99.93 \%$ of the total signal energy, meaning that the other six endmembers only occurs in a small percentage of the subimage.

A visual comparison between VCA results on the Cuprite dataset and the ground truth presented in [58] shows that the first component [see Fig. 16(a)] is predominantly Alunite, the second, third, sixth, and11th components [see Fig. 16(b), (c), (f), and (k)] are Kaolinite, the fourth component [see Fig. 16(d)] is Sphene, the fifth component [see Fig. 16(e)] is Andradite, the seventh component [see Fig. 16(g)] is predominantly Nontronite, the eighth component [see Fig. 16(h)] is Muscovite, the ninth and tenth components [see Fig. 16(i) and (j)] are Pyrope, the 12th component [see Fig. 16(1)] is Dumortierite, the 13th component [see Fig. 16(k)] is Montmorillonite, the 14th component [see Fig. 16(n)] is Buddingtonite.

In order to confirm the classification based on the estimated abundance fractions, a comparison of the estimated VCA endmember signatures with laboratory spectra [48] is presented in Fig. 17. The signatures provided by VCA are scaled by a factor in order to minimize the mean square error between them and the respective library spectra. The estimated signatures are close to the laboratory spectra. The larger mismatches occur for Andradite and Pyrope signatures, but only on a small percentage of the total bands.

Table II compares the spectral angles between extracted endmembers and laboratory reflectances for VCA, N-FINDR, and PPI algorithms. First column shows the laboratory substances with smaller spectral angle distance with respect to the signature extracted by VCA algorithm; Second column shows the respective angle. Third and fourth columns are as the second one, except when the closest spectral substance is different from the correspondent VCA one. In these cases, we write the name of the substance. The displayed results follow the pattern of behavior shown in the simulations, where VCA performs better than PPI and better or similarly to N-FINDR.

\section{CONCLUSION}

In this paper, we have proposed a new algorithm to unmix linear mixtures of hyperspectral sources, termed vertex component analysis. The VCA algorithm is unsupervised and is based on the geometry of convex sets. It exploits the fact that endmembers occupy the vertices of a simplex.

VCA algorithm assumes the presence of pure pixels in the data and iteratively projects data onto a direction orthogonal to the subspace spanned by the endmembers already determined. The new endmember signature corresponds to the extreme of the projection. The algorithm iterates until the number of endmembers is exhausted.

A comparison of VCA with pixel purity index [33] and N-FINDR [38] algorithms is conducted. Several experiments with simulated data lead to the conclusion that VCA performs better than PPI and better than or similarly to N-FINDR. VCA has, however, the lowest computational complexity among these three algorithms. Savings in computational complexity ranges between one and two orders of magnitude. This conclusion has great impact when the dataset has a large number of pixels. VCA was also applied to real hyperspectral data. The results achieved show that VCA is an effective tool to unmix hyperspectral data.

\section{REFERENCES}

[1] B. Hapke, Theory of Reflectance and Emmittance Spectroscopy. Cambridge, U.K.: Cambridge Univ. Press, 1993.

[2] R. N. Clark and T. L. Roush, "Reflectance spectroscopy: Quantitative analysis techniques for remote sensing applications," J. Geophys. Res., vol. 89, no. B7, pp. 6329-6340, 1984.

[3] T. Lillesand and R. Kiefer, Remote Sensing and Image Interpretation, 3rd ed. New York: Wiley, 1994.

[4] G. Vane, R. Green, T. Chrien, H. Enmark, E. Hansen, and W. Porter, "The Airborne Visible/Infrared Imaging Spectrometer (AVIRIS)," Remote Sens. Environ., vol. 44, pp. 127-143, 1993.

[5] M. O. Smith, J. B. Adams, and D. E. Sabol, "Spectral mixture analysis-New strategies for the analysis of multispectral data," in Imaging Spectrometry -A Tool for Environmental Observations, J. Hill and J. Megier, Eds. Brussels and Luxembourg, Belgium: ECSC, EEC, EAEC, 1994, pp. 125-143.

[6] A. R. Gillespie, M. O. Smith, J. B. Adams, S. C. Willis, A. F. Fisher, and D. E. Sabol, "Interpretation of residual images: Spectral mixture analysis of AVIRIS images, Owens Valley, California," in Proc. 2nd AVIRIS Workshop, vol. , R. O. Green, Ed., 1990, JPL Pub. 90-54, pp. 243-270.

[7] J. J. Settle, "On the relationship between spectral unmixing and subspace projection," IEEE Trans. Geosci. Remote Sens., vol. 34, no. 4, pp. 1045-1046, Jul. 1996.

[8] Y. H. Hu, H. B. Lee, and F. L. Scarpace, "Optimal linear spectral unmixing," IEEE Trans. Geosci. Remote Sens., vol. 37, no. 1, pp. 639-644, Jan. 1999.

[9] M. Petrou and P. G. Foschi, "Confidence in linear spectral unmixing of single pixels," IEEE Trans. Geosci. Remote Sens., vol. 37, no. 1, pp. 624-626, Jan. 1999

[10] S. Liangrocapart and M. Petrou, "Mixed pixels classification," in Proc. SPIE Conf. Image and Signal Processing for Remote Sensing IV, vol. 3500, 1998, pp. 72-83. 
[11] N. Keshava and J. Mustard, "Spectral unmixing," IEEE Signal Process. Mag., vol. 19, no. Jan., pp. 44-57, 2002.

[12] R. B. Singer and T. B. McCord, "Mars: Large scale mixing of bright and dark surface materials and implications for analysis of spectral reflectance," in Proc. 10th Lunar and Planetary Sci. Conf., 1979, pp. $1835-1848$.

[13] B. Hapke, "Bidirection reflectance spectroscopy. I. Theory," J. Geophys. Res., vol. 86, pp. 3039-3054, 1981.

[14] R. Singer, "Near-infrared spectral reflectance of mineral mixtures: Systematic combinations of pyroxenes, olivine, and iron oxides," J. Geophys. Res., vol. 86, pp. 7967-7982, 1981.

[15] B. Nash and J. Conel, "Spectral reflectance systematics for mixtures of powdered hypersthene, labradoride, and ilmenite," J. Geophys. Res., vol. 79, pp. 1615-1621, 1974

[16] C. C. Borel and S. A. Gerstl, "Nonlinear spectral mixing models for vegetative and soils surface," Remote Sens. Environ., vol. 47, no. 2, pp. 403-416, 1994.

[17] C.-I Chang, Hyperspectral Imaging: Techniques for Spectral Detection and Classification. New York: Kluwer, 2003.

[18] G. Shaw and H. Burke, "Spectral imaging for remote sensing," Lincoln Lab. J., vol. 14, no. 1, pp. 3-28, 2003.

[19] D. Manolakis, C. Siracusa, and G. Shaw, "Hyperspectral subpixel target detection using linear mixing model," IEEE Trans. Geosci. Remote Sens., vol. 39, no. 7, pp. 1392-1409, Jul. 2001.

[20] N. Keshava, J. Kerekes, D. Manolakis, and G. Shaw, "An algorithm taxonomy for hyperspectral unmixing," in Proc. SPIE AeroSense Conf. Algorithms for Multispectral and Hyperspectral Imagery VI, vol. 4049, 2000, pp. 42-63.

[21] A. S. Mazer and M. Martin et al., "Image processing software for imaging spectrometry data analysis," Remote Sens. Environ., vol. 24, no. 1 , pp. 201-210, 1988

[22] R. H. Yuhas, A. F. H. Goetz, and J. W. Boardman, "Discrimination among semi-arid landscape endmembres using the Spectral Angle Mapper (SAM) algorithm," in Summaries 3rd Annu. JPL Airborne Geoscience Workshop, vol. 1, R. O. Green, Ed., 1992, JPL Publ. 92-14, pp. 147-149.

[23] J. C. Harsanyi and C.-I Chang, "Hyperspectral image classification and dimensionality reduction: An orthogonal subspace projection approach," IEEE Trans. Geosci. Remote Sens., vol. 32, no. 4, pp. 779-785, Jul. 1994.

[24] C.-I Chang, X.-L. Zhao, M. L. G. Althouse, and J. J. Pan, "Least squares subspace projection approach to mixed pixel classification for hyperspectral images," IEEE Trans. Geosci. Remote Sens., vol. 36, no. 3, pp. 898-912, May 1998.

[25] D. C. Heinz, C.-I Chang, and M. L. G. Althouse, "Fully constrained least squares-based linear unmixing," in Proc. IGARSS, 1999, pp. 1401-1403.

[26] P. Common, C. Jutten, and J. Herault, "Blind separation of sources, part II: Problem statement," Signal Process., vol. 24, pp. 11-20, 1991.

[27] J. Bayliss, J. A. Gualtieri, and R. Cromp, "Analysing hyperspectral data with independent component analysis," Proc. SPIE, vol. 3240, pp. 133-143, 1997.

[28] C. Chen and X. Zhang, "Independent component analysis for remote sensing study," in Proc. SPIE Symp. Remote Sensing Conf. Image and Signal Processing for Remote Sensing V, vol. 3871, 1999, pp. 150-158.

[29] T. M. Tu, "Unsupervised signature extraction and separation in hyperspectral images: A noise-adjusted fast independent component analysis approach," Opt. Eng., vol. 39, no. 4, pp. 897-906, 2000.

[30] S.-S. Chiang, C.-I Chang, and I. W. Ginsberg, "Unsupervised hyperspectral image analysis using independent component analysis," in Proc. IGARSS, vol. 7, 2000, pp. 3136-3138.

[31] J. M. P. Nascimento and J. M. B. Dias, "Does independent component analysis play a role in unmixing hyperspectral data?," in Pattern Recognition and Image Analysis. ser. , F. J. Perales, A. Campilho, and N. P. B. A. Sanfeliu, Eds. Berlin, Germany: Springer-Verlag, 2003, vol. 2652, Lecture Notes in Computer Science, pp. 616-625.

[32] A. Ifarraguerri and C.-I Chang, "Multispectral and hyperspectral image analysis with convex cones," IEEE Trans. Geosci. Remote Sens., vol. 37, no. 2, pp. 756-770, Mar. 1999.

[33] J. Boardman, "Automating spectral unmixing of AVIRIS data using convex geometry concepts," in Summaries 4th Annu. JPL Airborne Geoscience Workshop, vol. 1, 1993, JPL Pub. 93-26, pp. 11-14.

[34] M. D. Craig, "Minimum-volume transforms for remotely sensed data," IEEE Trans. Geosci. Remote Sens., vol. 32, no. 1, pp. 99-109, Jan. 1994.

[35] C. Bateson, G. Asner, and C. Wessman, "Endmember bundles: A new approach to incorporating endmember variability into spectral mixture analysis," IEEE Trans. Geosci. Remote Sens., vol. 38, no. 2, pp. 1083-1094, Mar. 2000.
[36] R. Seidel, Convex Hull Computations. Boca Raton, FL: CRC, 1997, ch. 19 , pp. $361-375$.

[37] S. Geman and D. Geman, "Stochastic relaxation, Gibbs distribution and the Bayesian restoration of images," IEEE Trans. Pattern Anal. Mach. Intell., vol. 6, no. 6, pp. 721-741, Jun. 1984.

[38] M. E. Winter, "N-findr: An algorithm for fast autonomous spectral end-member determination in hyperspectral data," in Proc. SPIE Conf. Imaging Spectrometry V, 1999, pp. 266-275.

[39] J. Boardman, F. A. Kruse, and R. O. Green, "Mapping target signatures via partial unmixing of AVIRIS data," in Summaries 5th JPL Airborne Earth Science Workshop, vol. 1, 1995, pp. 23-26.

[40] J. Theiler, D. Lavenier, N. Harvey, S. Perkins, and J. Szymanski, "Using blocks of skewers for faster computation of pixel purity index," presented at the SPIE Int. Conf. Optical Science and Technology, 2000.

[41] D. Lavenier, J. Theiler, J. Szymanski, M. Gokhale, and J. Frigo, "FPGA implementation of the pixel purity index algorithm," presented at the Proc. SPIE Photonics East, Workshop on Reconfigurable Architectures, 2000.

[42] J. H. Bowles, P. J. Palmadesso, J. A. Antoniades, M. M. Baumback, and L. J. Rickard, "Use of filter vectors in hyperspectral data analysis," in Proc. SPIE Conf. Infrared Spaceborne Remote Sensing III, vol. 2553, 1995, pp. 148-157.

[43] J. H. Bowles, J. A. Antoniades, M. M. Baumback, J. M. Grossmann, D. Haas, P. J. Palmadesso, and J. Stracka, "Real-time analysis of hyperspectral data sets using NFL's orasis algorithm," in Proc. SPIE Conf. Imaging Spectrometry III, vol. 3118, 1997, pp. 38-45.

[44] J. M. Grossmann, J. Bowles, D. Haas, J. A. Antoniades, M. R. Grunes, P. Palmadesso, D. Gillis, K. Y. Tsang, M. Baumback, M. Daniel, J. Fisher, and I. Triandaf, "Hyperspectral analysis and target detection system for the Adaptative Spectral Reconnaissance Program (ASRP)," in Proc. SPIE Conf. Algorithms for Multispectral and Hyperspectral Imagery IV, vol. 3372, 1998, pp. 2-13.

[45] I. T. Jolliffe, Principal Component Analysis. New York: SpringerVerlag, 1986

[46] A. Green, M. Berman, P. Switzer, and M. D. Craig, "A transformation for ordering multispectral data in terms of image quality with implications for noise removal," IEEE Trans. Geosci. Remote Sens., vol. 26, no. 1, pp. 65-74, Jan. 1994.

[47] L. L. Scharf, Statistical Signal Processing, Detection Estimation and Time Series Analysis. Reading, MA: Addison-Wesley, 1991.

[48] R. N. Clark, G. A. Swayze, A. Gallagher, T. V. King, and W. M. Calvin, "The U.S. Geological Survey digital spectral library: Version 1: 0.2 to 3.0 m," U.S. Geol. Surv., Denver, CO, Open File Rep. 93-592, 1993.

[49] J. H. Bowles, M. Daniel, J. M. Grossmann, J. A. Antoniades, M. M. Baumback, and P. J. Palmadesso, "Comparison of output from orasis and pixel purity calculations," in Proc. SPIE Conf. Imaging Spectrometry IV, vol. 3438,1998 , pp. $148-156$

[50] A. Plaza, P. Martinez, R. Perez, and J. Plaza, "Spatial/spectral endmember extraction by multidimensional morphological operations," IEEE Trans. Geosci. Remote Sens., vol. 40, no. 9, pp. 2025-2041, Sep. 2002.

[51] — "A quantitative and comparative analysis of endmember extraction algorithms from hyperspectral data," IEEE Trans. Geosci. Remote Sens., vol. 42, no. 3, pp. 650-663, Mar. 2004.

[52] S. Kullback, Information Theory and Statistics, Peter Smith, London, U.K., 1978.

[53] C. Chang, "An information-theoretic approach to spectral variability, similarity, and discrimination for hyperspectral image analysis," IEEE Trans. Inf. Theory, vol. 46, no. 5, pp. 1927-1932, Aug. 2000.

[54] E. Kaltofen and G. Villard, "On the complexity of computing determinants," in Proc. 5th Asian Symp. Computer Mathematics, vol. 9, Lecture Notes Series on Computing, K. Shirayanagi and K. Yokoyama, Eds. Singapore, 2001, pp. 13-27.

[55] G. Swayze, R. Clark, S. Sutley, and A. Gallagher, "Ground-truthing aviris mineral mapping at Cuprite, Nevada," in Summaries 3rd Annu. JPL Airborne Geosciences Workshop, vol. 1, 1992, pp. 47-49.

[56] R. Ashley and M. Abrams, "Alteration mapping uing multispectral images-Cuprite Mining District, Esmeralda County," U.S. Geol. Surv., Denver, CO, Open File Rep. 80-367, 1980.

[57] M. Abrams, R. Ashley, L. Rowan, A. Goetz, and A. Kahle, "Mapping of hydrothermal alteration in the Cuprite Mining District, Nevada, using aircraft scanner images for the spectral region 0.46 to $2.36 \mathrm{~mm}$," $\mathrm{Ge}$ ology, vol. 5, pp. 713-718, 1977.

[58] G. Swayze, "The hydrothermal and structural history of the Cuprite Mining District, southwestern Nevada: An integrated geological and geophysical approach,” Ph.D. dissertation, Univ. Colorado, Boulder, 1997. 
[59] A. Goetz and V. Strivastava, "Mineralogical mapping in the cuprite mining district," in Proc. Airborne Imaging Spectrometer Data Analysis Workshop, 1985, JPL Pub. 5-41, pp. 22-29.

[60] F. Kruse, J. Boardman, and J. Huntington, "Comparision of EO-1 Hyperion and airborne hyperspectral remote sensing data for geologic applications," presented at the SPIE Aerospace Conf., 2002.

[61] C.-I Chang and Q. Du, "Estimation of number of spectrally distinct signal sources in hyperspectral imagery," IEEE Trans. Geosci. Remote Sens., vol. 42, no. 3, pp. 608-619, Mar. 2004.

[62] J. Harsanyi, W. Farrand, and C.-I Chang, "Determining the number and identity of spectral endmembers: An integrated approach using Neyman-Pearson eigenthresholding and iterative constrained rms error minimization," presented at the 9th Thematic Conf. Geologic Remote Sensing, 1993.

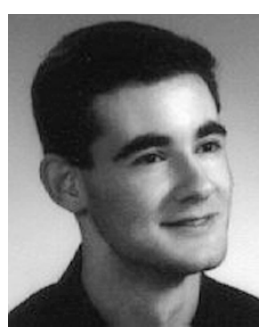

José M. P. Nascimento (S'03) received the B. S. and E.E. degree from Instituto Superior de Engenharia de Lisboa, Politechnic Institute of Lisbon, Lisbon, Portugal, and the M.Sc. degree in electrical and computer engineer from Instituto Superior Técnico (IST), Technical University of Lisbon, in 1993, 1995, and 2000 , respectively. He is currently pursuing the Ph.D. degree in electrical engineering at Instituto Superior Técnico, Technical University of Lisbon.

$\mathrm{He}$ is currently a Professor in the Department of Electronics, Telecommunications and Computer's Engineering, Instituto Superior de Engenharia de Lisboa. He is also Researcher with the Institute of Telecommunications. His research interests include remote sensing, signal and image processing, pattern recognition, and communications.

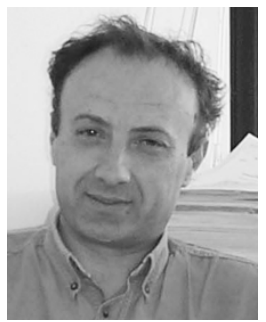

José M. Bioucas Dias (S'87-M'95) received the E.E., M.Sc., and Ph.D. degrees in electrical and computer engineering from the the Instituto Superior Técnico (IST), Technical University of Lisbon, Lisbon, Portugal, in 1985, 1991, and 1995, respectively.

He is currently an Assistant Professor with the Department of Electrical and Computer Engineering, IST. He is also a Researcher with the Communication Theory and Pattern Recognition Group, IST. His research interests include remote sensing, signal and image processing, pattern recognition, and communications. 